\title{
Consideraciones generales sobre las políticas públicas de regularización de asentamientos informales en América Latina
}

\author{
EDÉSIO FERNANDES ** \\ ** Miembro de la Teaching Faculty del Lincoln Institute of Land Policy, y de la Development Planning Unit de la \\ Universidad College London.
}

\begin{abstract}
This paper proposes a critical analysis on the growing tradition of implementing public policies for the regulation of informal settlements in urban areas, aiming at contributing to a more adequate treatment of informal urban development in Latin America. The paper starts with a general discussion on the main causes of the phenomenon, stressing the need for preventive public policies to provide formal access to urban land and housing. Based on this analysis and on the main lessons from the Latin American experience we discuss the nature of existing regulation programs. As a conclusion, the paper argues that it is necessary to re-think the dominant legalization policies so as to promote, in an integrated manner, both security of tenure and socio-spatial integration.
\end{abstract}

KEYWORDS: informal settlements, security of tenure, property rights regulation programs

RESUMEN: Este artículo propone algunas reflexiones críticas sobre la tradición creciente de implementación de políticas públicas de regularización de asentamientos informales en áreas urbanas, con el objetivo de contribuir para que el proceso de crecimiento informal de las ciudades latinoamericanas sea enfrentado de manera más adecuada. Después de discutir las principales causas del fenómeno de desarrollo urbano informal y de destacar la necesidad de políticas preventivas de acceso formal al suelo y a la vivienda, el artículo propone una reflexión sobre la naturaleza de los programas de regularización de asentamientos consolidados, basadas sobretodo en las lecciones de la experiencia latinoamericana. Como conclusión, el artículo argumenta que es necesario repensar las políticas de legalización dominantes de asentamientos para promover, de manera integrada, seguridad de la tenencia e integración socioespacial.

PALABRAS CLAVES: asentamientos informales, seguridad de la tenencia, derechos de propiedad, programas de regularización.

Recibido el 19 de diciembre de 2007, aprobado el 7 de julio de 2008.

Correspondencia: edesiofernandes@compuserve.com. 


\section{Introducción}

El proceso de urbanización viene registrando en períodos recientes tasas cada vez más aceleradas en Asia y África (Davis, 2006; Durand-Lasserve \& Royston, 2002; Neuwirth, 2005; Huchzermeyer \& Karam, 2006; Verma (2002), pero también en América Latina —región que desde hace mucho se ha consolidado como netamente urbanizada, con más de un $75 \%$ de su población viviendo en áreas urbanas - las tasas de crecimiento urbano siguen altas, aunque estén decreciendo. El patrón de urbanización en la región combina, históricamente, procesos de exclusión social y segregación espacial. En especial, un número creciente de latinoamericanos sigue adhiriendo a la informalidad: según estimaciones conservadoras, al menos uno de cada cuatro ciudadanos solamente tiene acceso a la tierra urbana y a la vivienda únicamente a través de procesos informales, generando una intensa proliferación de asentamientos informales en áreas urbanas. Como resultado, políticas y programas de regularización de asentamientos informales están siendo implementados en diversos países latinoamericanos.

Más que nunca, la producción informal de ciudad en Latinoamérica hoy está dejando de ser excepción para convertirse en regla. Aunque los datos sean siempre imprecisos, en ciudades como São Paulo y Río de Janeiro ya se admite oficialmente que el porcentaje de informalidad urbana se acerca al 50 ó 55\%, considerándose únicamente los procesos de acceso al suelo y a la vivienda, dejando por tanto a un lado otros procesos de informalidad urbana como la construcción irregular, los locales comerciales informales y otras prácticas. En otras ciudades brasileñas como Recife y Salvador de Bahía, la informalidad urbana alcanza magnitudes del orden de un $70 \%$ a un $80 \%$. Las cifras identificadas en ciudades de otros países de la región —Venezuela, Colombia, Ecuador, Perú, El Salvador y, más recientemente, también Argentina y Uruguay - indican igualmente que el proceso de producción informal del espacio urbano va en ascenso (Abramo, 2003; Cravino, 2006; Zeledón, 2006).

En este contexto, es necesario señalar que no se trata de un mero síntoma de un modelo de desarrollo económico y crecimiento urbano o de una simple distorsión de dicho modelo, sino del modelo en sí mismo, de la forma latinoamericana de producir espacio urbano, es decir, una producción de ciudad que se da, cada vez más, al margen de la ley, incumpliendo especialmente los estándares y criterios establecidos por la legislación civil y urbanística (Fernandes, 2005a).

Hay en Latinoamérica una gran diversidad de procesos informales que corresponden no sólo a las diferentes situaciones registradas dentro de cada ciudad o incluso de cada asentamiento informal, sino también una profunda heterogeneidad de situaciones de tenencia de la tierra identificadas en cada país. Las más comunes son seguramente las ocupaciones informales de áreas públicas y privadas, sea en favelas, villas, barriadas u otras formas de ocupación irregular, sea mediante la compra de lotes y/o de casas en parcelaciones "clandestinas", "piratas" e "irregulares". Existe, sin embargo, toda una serie de otros procesos informales menos conocidos - y, por ende, menos discutidos-, como son el aumento de densidad de ocupación de tugurios; el crecimiento de pequeños pueblos creados para dar soporte a las actividades agropecuarias, y que se transforman en verdaderas ciudades; la proliferación de subdivisión de lotes regulares, 
dando lugar a viviendas independientes, pero de tamaños exiguos; las ocupaciones a las orillas de los ríos que se van creando informalmente, etc.

$\mathrm{Si}$, por una parte, esta diversidad de procesos informales tiene como rasgos distintivos sus diferentes implicaciones jurídicas, por otra, lo que los une es la ausencia de infraestructura urbanística y ambiental adecuada, de equipamientos colectivos y de servicios públicos, así como la generalizada precariedad de lo edificado aunque, a lo largo de varios años, muchas de ellas logren alcanzar un relativo grado de consolidación.

Pese al enorme alcance y a las serias implicaciones socioeconómicas, jurídicas, políticas y ecológicas del proceso de producción informal de las ciudades latinoamericanas, por lo general, las respuestas del poder público — principalmente a través de programas de regularización de asentamientos informales, desde todos los ámbitos gubernamentales- son todavía pocas y poco satisfactorias. Si bien algunos países como el Perú y México han aprobado leyes específicas ya en las décadas del 60 y 70, cuando diversos estudios académicos abrieron la discusión sobre la cuestión de la informalidad urbana, la mayoría de experiencias de regularización de asentamientos informales en América Latina inició su andadura a partir de la década del 80, generando una tradición investigadora creciente.

Más recientemente, la discusión regional e internacional acerca de los programas de regularización alcanzó una relevancia aun mayor, convirtiéndose en objeto de la Campaña Global por la Seguridad en la Tenencia de la Vivienda, promocionada desde 1999 por UN-HABITAT. Diversos encuentros importantes sobre el tema se están realizando en diferentes países latinoamericanos por iniciativa de diversas organizaciones, destacándose en especial, el trabajo educativo pionero desarrollado por el Departamento de América Latina y el Caribe del Lincoln Institute of Land Policy.

Este artículo propone algunas reflexiones críticas sobre esta tradición creciente de implementación de políticas públicas de regularización de asentamientos informales, con el objetivo de contribuir para que el proceso de crecimiento informal de las ciudades latinoamericanas sea confrontado de manera más adecuada. Estas son reflexiones resultantes de las principales investigaciones internacionales sobre el tema del desarrollo informal, así como de mi experiencia personal trabajando con programas de regularización en diversos países y regiones hace más de 25 años.

\section{Deshaciendo algunos viejos mitos}

Aunque generalmente se les otorgue una gran importancia a los programas de regularización como forma de hacer frente al fenómeno del crecimiento de la pobreza urbana, existen innumerables discusiones y controversias sobre la naturaleza, posibilidades, problemas y perspectivas de dichos programas. En concreto, una característica de muchos países latinoamericanos es el hecho de que las tasas de crecimiento informal hoy en día son aun más significativas que las tasas de crecimiento de la pobreza social, lo que exige especial reflexión a la hora de formular políticas públicas de regularización de asentamientos informales. 
Existe, adicionalmente, otro mito que deshacer: el de que el problema del crecimiento urbano informal es una cuestión que aflige únicamente a las grandes ciudades. Los datos son todavía poco sistematizados, sin embargo, en Brasil por ejemplo, ya se cuenta con datos recientes que sugieren que este proceso de crecimiento intensivo informal de las ciudades ya se extendió tanto hacia las ciudades de porte mediano, como hacia las ciudades pequeñas. Es decir, es fundamental comprender que este proceso de producción informal es constituyente del espacio urbano latinoamericano, tratándose de proceso estructural que, como tal, no está aislado, no constituye una excepción, y no se trata de una cuestión exclusiva de las grandes ciudades (Fernandes, 2005b).

Por lo general, todavía no existe una comprensión adecuada acerca de las causas, dinámicas e implicaciones de los procesos de producción de la informalidad urbana, inclusive en lo que se refiere a las formas de acceso a la tierra urbana y a la vivienda. Ello viene provocando problemas y discusiones de diversa consideración, al punto que son muchos los que defienden la idea de que — tal como han sido formulados e implementados - los programas de regularización, en lugar de promocionar la necesaria integración socioespacial, acaban por mantener y agudizar el status quo que originalmente provocó la informalidad urbana en las ciudades latinoamericanas.

En este contexto, es crucial reflexionar amplia y críticamente acerca de los programas de regularización; por una parte, porque no es necesario inventar la rueda a cada día; por otra, para permitir que los equívocos ya cometidos no se repitan, sobre todo por aquellos países y ciudades que formulan por primera vez sus programas de regularización. Con más de treinta años de experiencias acumuladas, América Latina tiene mucho que contribuir para el importante debate internacional, especialmente en lo que se refiere a la búsqueda de caminos innovadores que permitan a los gobiernos y a la sociedad enfrentarse con la cuestión del crecimiento urbano informal.

Otro mito que hay que poner en tela de juicio en este debate es el de que la informalidad o ilegalidad en la producción del espacio urbano se vincula únicamente a los sectores sociales de bajos niveles de ingreso. De hecho, son muchos los procesos y prácticas de informalidad e ilegalidad urbanas entre los grupos privilegiados de la sociedad latinoamericana. Sin embargo, el énfasis puesto en la discusión de los asentamientos informales de grupos sociales pobres corresponde a la urgencia de formulación de políticas públicas para su enfrentamiento, dadas las implicaciones de todo orden que afectan no sólo a estos grupos, en forma directa, sino al conjunto de la ciudad.

Entre los impactos y costos sociales del crecimiento informal se destacan el aumento de la exclusión social y de la segregación espacial, así como la creciente y perversa asociación entre habitantes de asentamientos irregulares y criminales, dando lugar a una serie de políticas públicas represivas y reacciones negativas, colmadas de prejuicios de parte de la opinión pública. Entre los problemas jurídicos con relación a la falta de seguridad individual de la tenencia de la tierra, hay que mencionar, además de la vulnerabilidad de los residentes frente a las acciones y políticas de desalojo forzado, también la falta de acceso amplio de los habitantes de asentamientos informales al crédito formal, así como, muy frecuentemente, la falta 
de reconocimiento de derechos básicos, como es disponer de una dirección. De hecho, son millones los latinoamericanos que no disponen de una dirección oficial en la que recibir su correspondencia, lo que, entre otros factores, induce a la discriminación sistemática de estas personas a la hora de ingresar al mercado laboral.

El crecimiento informal de las ciudades también implica una mayor vulnerabilidad política de estos grupos sociales frente a las prácticas tradicionales de clientelismo y mantenimiento de intereses conservadores que hacen de las ciudades palco de la acumulación del capital, sin más consideración de otros valores sociales, colectivos, culturales y ambientales. Es igualmente importante destacar que el proceso de crecimiento informal implica costos económicos muy altos para todos, incluso para aquellos que no viven en esta condición. En gran medida producida informalmente, la ciudad latinoamericana es extremadamente costosa, en virtud de la irracionalidad administrativa y de su ineficiencia económica; de los altos costos de implantación de servicios y equipamientos; de la fragmentación urbana, así como de la degradación ambiental. Adicionalmente, la regularización de asentamientos informales consolidados implica altos costos, tanto por tratarse de un proceso que consume mucho tiempo, como porque requiere inversiones masivas, alcanzando montos superiores a los necesarios a la implantación de políticas de naturaleza preventiva.

Existe una percepción generalizada de que aquellos que viven en asentamientos informales lo hacen por ausencia de cualquier otra alternativa. Ello puede ser cierto en el sentido en que ni los mercados, ni las políticas de vivienda, ofrecen soluciones adecuadas de acceso al suelo y a la vivienda — suficientes y asequibles — para estos grupos. Entretanto, ello no supone que esta "única opción” sea buena desde las ópticas urbanística y ambiental. De hecho, lo que se observa con frecuencia creciente son personas viviendo precariamente en áreas de riesgo, en áreas de protección a manantiales, en áreas de preservación permanente, o en otras áreas públicas. Sin embargo, también desde el punto de vista de los pobres que viven en asentamientos ilegales hay altos costos económicos que considerar. Así todo, otro dato debe sumarse a esta consideración: investigaciones recientes demuestran que el precio del metro cuadrado producido informalmente en las ciudades latinoamericanas es altísimo (Abramo, 2003). En términos relativos, los habitantes de asentamientos informales pagan precios absurdos para vivir en condiciones extremadamente precarias. Se creó toda una industria alrededor de la producción informal de la ciudad, que no se relaciona únicamente con el aumento de la pobreza.

De hecho, hay un conjunto de otras cuestiones y factores que hacen que este patrón excluyente no sólo se perpetúe, sino que se amplíe. Definitivamente, es fundamental enfrentar este proceso de crecimiento de la informalidad urbana porque, aunque desde el punto de vista de los habitantes este proceso pueda ofrecer soluciones inmediatas, dichas soluciones están lejos de lo ideal, tanto para los propios habitantes, como para el conjunto de las ciudades. Así, hay que conocer debidamente las causas de este proceso para que se las pueda enfrentar.

\section{Las causas del proceso}

Tradicionalmente, se afirma que la informalidad urbana deriva de la incapacidad estructural del poder público de garantizar la oferta de lotes con servicios en función de la escasez de 
alternativas de vivienda, sean estas ofrecidas por el poder público (debido a la inexistencia de políticas habitacionales adecuadas), sean las producidas por los mercados formales, que son cada vez más esencialmente especulativos. A estas causas más frecuentemente discutidas, hay que añadir otras tres, señalándose las estrechas vinculaciones entre ellas.

En primer lugar, es necesario empezar a reconsiderar la tradición elitista y tecnócrata de la planificación urbana que se practica en Latinoamérica. En gran medida, la producción informal de las ciudades se da en función de la incidencia de criterios elitistas de planificación urbana que pueden ser técnicamente ideales, pero que no reflejan las condiciones socioeconómicas de producción de la ciudad, excluyendo por esta vía gran parte de la población y reservando para los pobres aquellas áreas que los mercados no tienen cualquier interés, o aquellas a las que no tienen acceso, como son las áreas de preservación y todo tipo de áreas inadecuadas para la edificación.

En segundo lugar, hay que destacar que muchas de estas leyes urbanísticas no son pasibles de implantación y fiscalización por las municipalidades, puesto que no tienen en cuenta la precaria capacidad de acción de los municipios, estando por tanto condenadas al incumplimiento. Este es el caso de muchas subdivisiones irregulares: con frecuencia, el precio del lote irregular es más o menos el mismo de la parcela regular, sin embargo los promotores inmobiliarios son conducidos a la informalidad o "prefieren" hacer la subdivisión irregular porque es más fácil, considerándose la enorme burocracia exigida. En muchas ciudades, se tarda de tres a cinco años para aprobar un proyecto de subdivisión, lo que hace con que la "opción” por la informalidad sea cada vez más frecuente. Claro está que todo ello tiene lugar en un contexto de falta de fiscalización y de represión, aliado a la preponderancia de sistemas políticos clientelistas que vienen fomentando y manipulando los procesos de producción informal de las ciudades.

En tercer lugar, es también fundamental que se comprenda que la ilegalidad urbana en América Latina se produce, en gran medida, por efecto del propio orden jurídico más amplio. La ley se constituye en un factor importante de la ampliación de la ilegalidad en la región, sobre todo porque todavía prevalece muy fuerte, dominante y hegemónica, una visión conservadora, individualista, civilista y patrimonialista de los derechos individuales de propiedad. Visión esta que sigue orientando gran parte de la acción de los tribunales e incluso de la opinión pública.

Enfrentar el proceso de producción informal de la ciudad implica enfrentar todas estas causas.

\section{La naturaleza de los programas de regularización}

Entonces, ¿cómo el poder público y la sociedad reaccionan y ofrecen respuestas a este proceso? La verdad es que no hay una respuesta única, las respuestas — bajo la forma de políticas propuestas y adoptadas - evolucionan a lo largo del tiempo, reflejando la compleja serie de relaciones existentes entre lo formal y lo informal, lo legal y lo ilegal, que son meras categorías analíticas, utilizadas con el sentido de marcar las diferencias entre procesos que son intrínsecamente interrelacionados. De hecho, no existe propiamente una "ciudad informal" que se produzca totalmente fuera del contexto de la legalidad, sino debates, contextos, diálogos 
contradictorios, dialécticos e íntimos entre el orden formal y el orden informal, entre legalidad y la ilegalidad (Fernandes y Varley, 2004).

Hasta la década del 70, la política dominante en la región era la misma que en la actualidad se constituye en la política oficial común a los contextos asiático y africano, es decir, la expulsión y/o remoción forzosa de los habitantes de asentamientos informales. A partir de la década del 80 , en algunas ciudades, se procedió a remociones colectivas que, por lo general, fueron mal sucedidas, si no rotundos fracasos. A partir de este punto se comienza a formular un nuevo concepto de enfrentamiento del problema del crecimiento informal de las ciudades, a través de la aprobación de políticas de regularización, es decir, de la propuesta de inclusión e integración de las poblaciones y de las áreas informales consolidadas, tanto en la estructura, como en la sociedad urbana (Fernandes, 2003a).

Antes de avanzar en esta discusión, es necesario señalar que programas de regularización tienen una naturaleza esencialmente curativa. Ellos se plantean como respuesta a realidades constituidas a lo largo del tiempo, situaciones consolidadas, en las cuales muchas veces también derechos se constituyeron. Dichos programas sólo tienen sentido y eficacia si concebidos en el contexto de un conjunto más amplio de políticas públicas preventivas, que rompan el círculo vicioso — y viciado - que, a lo largo de décadas, produjo ciudades por la vía de la informalidad.

En este sentido, programas de regularización tienen que, necesariamente, estar combinados con nuevas opciones de vivienda de interés social construidas por el poder público — desde todos los ámbitos administrativos - asociados a la apertura de nuevas líneas de crédito, sobre todo para la población más pobre, tradicionalmente excluida del acceso al crédito oficial. Otro dato fundamental es que, aun con las frecuentes menciones al déficit habitacional de los países latinoamericanos, apenas hay referencia al gran número de inmuebles vacantes en las áreas urbanas, tanto de propiedad privada, como de propiedad pública. Además, hay que enfrentar la cuestión de las tierras urbanas vacantes, con el sentido de otorgar una función social a los predios urbanizados - y, sin embargo, ociosos - para fines de política habitacional y de regularización. El hecho es que los centros de las grandes ciudades latinoamericanas se están vaciando, dejando ociosa una serie de áreas que se podrían ocupar con vivienda de interés social.

Asimismo, hay que reconocer que, en todos los niveles gubernamentales, aun el poder público más competente, más ágil y más bien intencionado es incapaz de hacerse cargo, por sí solo, de la solución del problema habitacional, en función de la enorme escala de la cuestión, acumulada a lo largo de décadas. De ahí se desprende la necesidad de una otra forma de acción: atraer al sector privado y al sector comunitario para que participen de la producción de tierra urbanizada para los sectores de bajos niveles de ingreso. La construcción de la vivienda en sí no es el gran problema latinoamericano, sí lo es el acceso a la tierra urbanizada, bien ubicada y dotada de servicios. Con este sentido, el Estatuto de la Ciudad en Brasil y la Operación Nuevo Usme en Colombia, entre otros avances y otras leyes urbanísticas importantes, ya indican una serie de elementos y de posibilidades para que el sector privado y el sector comunitario encuentren atractivo en la producción regular de la tierra urbanizada de naturaleza social (Rincón, 2005; Maldonado, 2006; Fernandes, 2003b). 
Así, el gran dilema de las ciudades latinoamericanas es el de cómo promocionar políticas y programas de regularización que sean sostenibles, combinando las dimensiones urbanística, ambiental, social y jurídica, lo que depende de una acción urgente y articulada del poder público en todos sus ámbitos administrativos.

\section{El derecho a la regularización}

Un problema creciente es que, exactamente en función de la omisión o ineficiencia de la acción del poder público a lo largo de décadas de ocupación informal, en muchos países los caminos judiciales están cada vez más abiertos para la proposición de acciones individuales o colectivas de parte de los habitantes de asentamientos informales que desean ver sus derechos formalmente reconocidos. Ya no se puede tratar la cuestión de la regularización tan solo como una cuestión técnica que esté exclusivamente dentro del ámbito de la acción discrecional del poder público: hay que reconocer que derechos subjetivos se están constituyendo en el tiempo.

En realidad, ya no hay que oponer valores externos a derechos, puesto que, en la actualidad, el orden jurídico de diversos países latinoamericanos consagra el derecho colectivo a la regularización de los asentamientos informales, sea por fuerza de las constituciones y leyes nacionales, sea por fuerza de los tratados internacionales firmados por estos países. De hecho, hasta fechas recientes, los programas de regularización se apoyaban sobre argumentos morales, humanitarios y sociopolíticos, así como religiosos. En paralelo, hay también que considerar un poderoso argumento de orden económico, que se encuentra muy bien sintetizado en la obra del economista peruano Hernando de Soto, que influyó grandemente la discusión y la formulación de políticas de regularización en distintos países (de Soto, 1986; 2000). Sin embargo, es necesario comprender que además, existe hoy un también fuertísimo argumento jurídico para justificar la formulación e implementación de programas de regularización.

Ello significa que el poder público en América Latina tiene, en estos momentos, 'la soga al cuello’ si quiere promocionar la regularización en su sentido más amplio, combinando la regularización urbanística de asentamientos y parcelas (con implantación de infraestructura, equipamientos y servicios) con la regularización ambiental, la regularización social (a través de programas de generación de empleo y renta), y la regularización jurídica, es decir, la aprobación de las parcelaciones informales y el reconocimiento de las parcelas, seguida de la titulación de los ocupantes. Caso sea de su interés actuar de esta manera amplia y articulada, el poder público debe actuar con urgencia, porque de no hacerlo, se expondrá al riesgo de ser "atropellado", como de hecho viene ocurriendo, por la vía judicial del reconocimiento de tan solo derechos a la titulación, en cuya hipótesis prevalece el concepto de regularización jurídica, stricto sensu.

\section{Objetivos de los programas de regularización}

Tradicionalmente, los programas de regularización declaran como objetivo garantizar la seguridad de la tenencia de la tierra. Con este mismo objetivo, hay una serie de campañas, programas y acciones en marcha, promocionados por agencias internacionales como UN-HABITAT, el Banco Mundial y los bancos regionales de desarrollo. Una de las llamadas Metas del Milenio, 
que orientan la liberación de gran parte de los recursos de las agencias internacionales, es disminuir la pobreza social, y uno de los criterios de verificación de la pobreza, de acuerdo a estas mismas agencias, es exactamente la (in)seguridad de la tenencia. Sin embargo, por mucho que el movimiento creciente de ideas y acciones que en estos momentos están en marcha en el escenario internacional sea de enorme importancia, la verdad es que aún se está trabajando a escalas muy tímidas, gotas en el océano. La iniciativa Cities Alliance, liderada por el Banco Mundial, por ejemplo, trabaja con el objetivo de reducir en 100 millones el número de personas que viven en condiciones precarias hasta el 2020, mientras que hoy se reconoce en el mundo en desarrollo cerca de un billón de personas viviendo informalmente en favelas o áreas semejantes. Referirse a 100 millones de personas, en este contexto, es una mínima gota de un proceso mucho más amplio.

Otro objetivo muy propio de las políticas de regularización en diversas ciudades latinoamericanas, al menos nominalmente, es la idea de que estos programas deban promover la integración socioespacial de las áreas y comunidades. La seguridad de la tenencia de la tierra es siempre una garantía de protección en contra del desalojo o remoción forzosa, objetivo que no puede ser subestimado, considerándose que, al tiempo que la adopción de políticas de regularización goza de progresivamente más aceptación, todavía existen muchas situaciones de desalojo y remoción en diversos países latinoamericanos. Sin embargo, seguridad de la tenencia de la tierra hoy en América Latina es un concepto que involucra a una serie de otros objetivos, más allá de la protección en contra del desahucio: acceso a crédito formal, producción de asentamientos sostenibles, reconocimiento de derechos de ciudadanía, fortalecimiento de organizaciones sociales, reconocimiento de los derechos de las mujeres, etc.

En especial, la dimensión de género de los programas de regularización necesita ser mejor comprendida y asimilada, porque las investigaciones muestran que mujeres y niños sufren mucho más el impacto de la producción informal de las ciudades, una vez que son ellas las que permanecen sobre el terreno, mientras que los varones pasan. En la actualidad, en muchas grandes ciudades, más de un $50 \%$ de los jefes de hogar son mujeres, y esta dimensión de género ya no puede ser ignorada si los programas de regularización efectivamente visan promover la integración y garantizar la permanencia de las poblaciones en los lugares que habitan.

\section{Lecciones de la experiencia de Latinoamérica}

Una primera lección a ser reconocida es que la experiencia acumulada en Latinoamérica a partir de sus programas de regularización ha sido un gran fracaso, sobre todo porque los objetivos declarados — de reconocimiento de la seguridad de la tenencia de la tierra y de promoción de la integración socioespacial— no se están traduciendo debidamente en instrumentos técnicos, jurídicos, financieros y urbanísticos adecuados. Por lo general, los mecanismos de gestión de tales programas son inadecuados o inexistentes. Tampoco existen los procesos sociopolíticos y socioeconómicos necesarios para que estos programas surtan efecto y se combinen con políticas preventivas, dada su naturaleza netamente curativa. Faltan igualmente procesos financieros, institucionales, de planificación y de soporte. 
Es en este contexto que, con frecuencia, diversos analistas concluyen que en lugar de promover integración socioespacial, estos programas acabarían por agudizar los procesos de segregación y las condiciones de producción informal de la ciudad, y que los recursos públicos se estarían empleando sin que se obtenga el debido retorno. Hay una serie de otras críticas sobre los efectos perversos de dichos programas, que, en su conjunto, comprueban la necesidad de comprensión de las causas del fenómeno y de la naturaleza de los programas, así como de la necesidad de su conciliación con políticas públicas más amplias, para que los programas de regularización puedan, efectivamente, contribuir para la democratización del acceso a la tierra y la vivienda (Fernandes y Smolka, 2004).

Otro aspecto que queda claro en la experiencia latinoamericana es que no se verifica cualquier relación directa, automática, entre programas de regularización — aun aquellos considerados exitosos - y la erradicación, o al menos reducción significativa de la pobreza social. El enfrentamiento de la pobreza requiere todo un conjunto articulado de acciones, de programas, de inversiones, de políticas de generación de renta y empleo. Naturalmente, un programa de regularización bien estructurado produce impactos positivos en el cotidiano de los habitantes; por ejemplo, la mujer que ya no necesita caminar 40 minutos para abastecerse de agua, y puede aprovechar este tiempo para coser en casa, generando algún tipo de ingreso para la familia. Sin embargo, y sin quitarles mérito, estas conquistas no son estructurales desde el punto de vista de la reducción de la pobreza.

Además, los programas de regularización, cuando existen (y no son muchos), todavía se conciben de manera aislada, sectorial y marginal en el contexto de las políticas públicas, como si estuviesen tratando de una situación excepcional. Son raros los municipios y gobiernos centrales que traen esta discusión sobre el enfrentamiento de la irregularidad para el corazón de la planificación urbana, de las leyes urbanísticas y planes municipales. Por lo general, las políticas de regularización son específicas, no llevando en cuenta el carácter estructural y estructurador de los procesos de producción informal del espacio urbano.

\section{La cuestión de la legalización}

Un aspecto crucial de la discusión internacional acerca de los programas de regularización se refiere a la cuestión de la legalización de los asentamientos y sus ocupantes. Un gran énfasis sobre la necesidad de distribuir títulos se ha planteado, habiendo una presión internacional de parte del Banco Mundial, del Banco Interamericano de Desarrollo y de otras agencias con el sentido de "legalizar lo ilegal", de formular programas de legalización y de distribuir títulos, lo que se viene haciendo en larga escala en diversos países y ciudades. Es importante destacar que, cuando se habla de títulos, tradicionalmente se está pensando tan sólo en una forma específica de legalización, es decir, el reconocimiento de títulos individuales de propiedad plena.

En gran medida, esta discusión internacional se basa en las mencionadas ideas de Hernando de Soto, para quien el gran reto planteado hoy para los países en desarrollo es garantizar el acceso al crédito, lo que sería posible a través de programas de legalización bajo la forma de la propiedad individual plena de los asentamientos informales, haciendo que los ocupantes, finalmente seguros de su tenencia, inviertan en el mejoramiento de casas y negocios informales. 
Con base en esta suposición, programas de legalización en masa se discutieron y se llevaron a la práctica en el Perú, El Salvador, Rumania, Filipinas, Egipto, etc. Se trata de una de las más polémicas discusiones de la actualidad (Fernandes, 2002).

El argumento de De Soto requiere discusión en profundidad, puesto que los análisis disponibles acerca de las experiencias acumuladas indican que el fenómeno es mucho más complejo de lo que De Soto deja entrever. El análisis del caso peruano, por ejemplo, indica que —a pesar del masivo proceso de privatización del patrimonio público, y en ausencia de cualquier preocupación sistemática con las dimensiones urbanística, ambiental y social durante el proceso de regularización-, se generaron áreas legalizadas, pero no sostenibles, es decir, sin servicios, transportes o equipamientos adecuados. Tampoco se materializó el prometido acceso al crédito oficial. Así, la seguridad individual de los habitantes puede haber sido plenamente alcanzada, puesto que están protegidos contra acciones de desalojo y procesos de remoción forzosa. Sin embargo, no se puede decir que se haya logrado una integración socioespacial de estas personas y áreas (Calderón, 2006; Zeledón, 2006).

En otras situaciones, sobre todo en áreas centrales, la mera legalización formal "a la De Soto" viene produciendo el efecto de amenazar la propia permanencia de los habitantes en el sitio original, ya que toda la inversión pública en el área regularizada puede ser apropiada por otras personas o agentes — con frecuencia, aquellos mismos agentes responsables de la producción informal del espacio- - ya que la privatización de áreas públicas puede estar asociada a procesos de "expulsión por el mercado" u otras fuerzas como el narcotráfico.

Aun reconociendo la importancia del título por una serie de motivos, ya se percibe una aceptación creciente de que no es el título el elemento que induce a las personas a realizar inversiones en sus casas y locales comerciales. En realidad, muchos asentamientos informales en ciudades latinoamericanas se consolidan con enorme celeridad: un día es un trozo de lata; al día siguiente, un ladrillo; de ahí a un mes, ya es una casa y una comunidad entera. Ello se deriva de la existencia de un conjunto de factores sociales, políticos e institucionales que generan una amplia percepción de seguridad, un pacto social reconocido como suficiente para garantizar que los individuos comiencen a invertir en la mejora de sus viviendas.

Por tanto, los títulos son importantes por una serie de motivos: seguridad individual de la tenencia de la tierra; conflictos con propietarios; conflictos domésticos; problemas de herencia; problemas de demarcación de tierras; presiones externas; tráfico de drogas; presiones del mercado, etc. La cuestión crucial es cómo conciliar la titulación con el objetivo de la integración socioespacial.

Con este sentido, el planteamiento que se viene reafirmando más frecuentemente es el que sigue: la obligación del Estado no es la de distribuir propiedad, es más, un mero programa de regularización no se constituye en instrumento apto para alterar la estructura concentrada e injusta de propiedad de la tierra. No se debe esperar que la regularización provoque cualquier revolución de la propiedad... Se trata apenas de una forma de reconocer el derecho social a la vivienda, que no puede reducirse al derecho de propiedad, menos aún al derecho individual de propiedad plena. Derecho a la vivienda es un concepto mucho más amplio, que ofrece 
un abanico de alternativas jurídico-políticas para la acción gubernamental, más allá de la formulación de programas de regularización. Opciones que pueden variar desde la propiedad individual plena, si se da el caso, a la propiedad colectiva, y que puede ser adquirida por la vía del procedimiento de usucapión (o prescripción) individual o colectivo, o a través de compra o donación; pero que también incluyen diversas formas de propiedad restringida como, por ejemplo, en el caso de la concesión del derecho real de uso o de la concesión de uso especial para fines de vivienda individual o colectiva.

También hay que considerar los contratos de alquiler social, una vez que, en realidad, no hay una única experiencia internacional de enfrentamiento de la crisis urbana y habitacional que no haya involucrado esta opción. Otras formas más precarias como permisos, autorizaciones de ocupación también deben considerarse. La dificultad reside en cómo elegir la mejor opción, habiendo ahí una serie de factores a considerar: los objetivos del programa de regularización, la situación jurídica existente, el régimen de propiedad, si el área es pública o privada, las costumbres y valores culturales y sociales, etc. (Payne, 2002).

No se trata, por tanto, de legalizar sin tener en cuenta otras preocupaciones urbanísticas y socioambientales, o intentar "atar las personas al suelo" impidiendo que las parcelas legalizadas se vendan. Se trata antes de reservar suelo urbano para la función de vivienda de interés social. Se trata de preservar aquellas áreas urbanizadas por iniciativa pública y que implicaron inversiones altísimas, para beneficiarios finales pertenecientes a los grupos sociales de bajos niveles de ingreso, o sea, los pobres de la ciudad en general, no necesariamente aquellos mismos pobres que ya vivían en el área. Si el habitante así lo desea, puede vender su casa para lanzarse a la economía informal, no hay mucho que se pueda hacer para impedirlo. Más importante es garantizar que el área regularizada siga ejerciendo su función social.

Hasta el día en que exista en los países latinoamericanos un contexto estructural de distribución de la renta y de la riqueza más justo, no se podrá echar mano de este tipo de protección social y jurídica. La cuestión es, entonces, garantizar el acceso a la tierra y garantizar que la tierra urbanizada se mantenga como área destinada al ejercicio de la función de vivienda social. Considerado de manera aislada, el título individual, por otra parte, termina muchas veces por conducir a efectos perversos. Si este título no está asociado a una serie de otros factores jurídicos y urbanísticos, puede generar una gran presión del mercado sobre las áreas urbanizadas y legalizadas, llevando a los habitantes originales a vender sus parcelas, permitiendo que otros agentes se apropien de ellas.

En este contexto, la otra gran lección de las experiencias latinoamericanas es que no es suficiente pensar la cuestión de la regularización apenas desde el punto de vista de los derechos individuales de los habitantes en asentamientos informales, es decir, de la seguridad individual de la tenencia: es necesario pensar cómo estos programas - combinando estrategias de planificación urbana y procesos de gestión democrática — pueden también garantizar la integración socioespacial. 


\section{Conclusión}

El reto de promover la regularización sostenible de los asentamientos informales está planteado especialmente para todos los países y ciudades latinoamericanos. Sobre todo, se trata de un enorme desafío dirigido a la sociedad latinoamericana.

Ya no se puede evitar el enfrentamiento de este problema galopante de la producción informal de la ciudad, con todas sus nefastas implicaciones para todos, no sólo para los habitantes de asentamientos informales, sino para las ciudades y para la sociedad urbana en su conjunto.

No hay respuestas sencillas, no hay respuestas únicas, no hay respuestas rápidas y no hay respuestas baratas... Como los recursos son pocos, tenemos todos que comprender mejor los procesos de producción de la informalidad urbana para mejor enfrentarlos.

\section{Referencias bibliográficas}

Abramo, P. (Org.) (2003). A Cidade da informalidade. O desafio das cidades latino-americanas. Rio de Janeiro: Sette Letras.

Calderón, J. (2006). Mercado de tierras urbanas, propiedad y pobreza. Lima: Lincoln Institute of Land Policy/SINCO Editores.

Cravino, M. C. (2006). Las villas de la ciudad. Mercado e informalidad urbana. Los Polvorines: Universidad Nacional del General Sarmiento.

Davis, M. (2006). Planet of slums. London y New York: Verso.

De Soto, H. (1986). El otro sendero: la revolución informal. México D.F.: Diana.

De Soto, H. (2000). El misterio del capital. Por qué el capitalismo triunfa en Occidente y fracasa en el resto del mundo. Lima: El Comercio.

Durand-Lasserve, A. \& Royston, L. (Orgs.) (2002). Holding their ground. Secure land tenure for the urban poor in developing countries. London: Earthscan.

Fernandes, E. \& Smolka, M. (2004). Land regularization and upgrading programs revisited. Land Lines, 16.

Fernandes, E. \& Varley, A. (Orgs.) (2004). Ciudades ilegales. La ley y el urbanismo en paises en desarrollo. Cochabamba: Promesha IIA.

Fernandes, E. (2002). The influence of de Soto's. The Mystery of Capital. Land Lines, 14, 5-8.

Fernandes, E. (2003a). Tenencia de la tierra y pobreza urbana en América Latina. Vivienda Popular, 12, 5-16

Fernandes, E. (2003b). Del Código Civil al Estatuto de la Ciudad: algunas notas sobre la trayectoria del Derecho Urbanístico en Brasil. EURE, 29, 87, 63-78.

Fernandes, E. (2005a). Límites y posibilidades de la democratización del acceso al suelo urbano y a la vivienda en Brasil después del Estatuto de la Ciudad. Ciudad y Territorio - Estudios Territoriales, 27, 146, 707-723.

Fernandes, E. (2005b). Aspectos jurídicos de los programas de regularización de la tenencia de la tierra urbana en Latinoamérica. En A. Patino (Org), Espacios urbanos no con-sentidos. Legalidad e ilegalidad en la producción de la ciudad, Colombia y Brasil. Medellín: Universidad Nacional de Colombia. 
Huchzermeyer, M. \& Karam, A. (Orgs.) (2006). Informal settlements: a perpetual challenge? Cape Town: UCT Press/Cordaid.

Maldonado, M. M. (2006). El proceso de construcción del sistema urbanístico colombiano: entre reforma urbana y ordenamiento territorial. En E. Fernandes \& B. Alfonsín (Orgs.), Direito Urbanístico. Estudos Brasileiros e Internacionais. Belo Horizonte: Del Rey.

Neuwirth, R. (2005). Shadow cities. New York: Routledge.

Payne, G. (Org.) (2002). Land, rights and innovation. Improving tenure security for the urban poor. London: ITDG.

Rincón, A. (Org.) (2005). Espacios urbanos no con-sentidos. Legalidad e ilegalidad en la producción de la ciudad. Colombia y Brasil. Medellín: Universidad Nacional de Colombia.

Verma, G. D. (2002). Slumming India. New Delhi: Penguin.

Zeledón, A. (2006). Reflexiones en torno al marco jurídico de El Salvador. En E. Fernandes \& Alfonsín, B. (Orgs), Direito Urbanístico. Estudos Brasileiros e Internacionais. Belo Horizonte: Del Rey. 